\title{
Application of Jiangxi intangible cultural heritage in modern fashion design
}

\author{
LiuYongru
}

\author{
Nanchang Institute of Science \& Technology, Nanchang 330108,China
}

Key words: intangible culture; fashion design; Nuo clothing; Hanfu costume design

\begin{abstract}
The Hanfu is Han inherits traditional costumes, dress is also important in the history of Jiangxi, which Jiangxi endemic to the clothing clothing type, associated with the local customs, especially with apparel modeling and process design is quite mature in Nanfeng County, Jiangxi is the fashion of intangible cultural heritage. These clothes are also the symbol of Chinese traditional culture, and they should be passed on and carried forward, which needs to be integrated into modern fashion design. This study will Hanfu clothing collar into the Nuo interchange of modern fashion design, provides a new reference for the modern fashion design creative.
\end{abstract}

\section{Introduction}

In Jiangxi fashion design history, it not only occupies the important position of Hanfu, also has the obvious local characteristics of Nuo dance clothing, the clothing heritage has a long history, but it has strong vitality, which belongs to the intangible cultural heritage. At present, the clothing has not been completely eliminated, in some important ceremonies and celebrations, such as clothing and Hanfu Nuo dance often appear. With the arrival of twenty-first Century, the clothing designers began to examine their own excellent part of traditional culture, many people began to study the intangible cultural heritage of modern design elements of traditional clothing, and through the promotion of the essence of traditional culture, the intangible cultural heritage revival, bring new vigor and vitality to modern fashion design.

\section{The overview of intangible cultural heritage}

Non material heritage is a non material things, then he is closely linked with people's life, and is a kind of cultural and artistic heritage, in oral interpretation and folk custom etiquette, is a form of traditional handicraft practice, related to the natural universe or the practice of traditional culture. Intangible cultural heritage is human centered, it has certain standard verification, such as the time of heritage and lineage, inheritance time must be in school or master son, being inherited over three generations, the overall time should be more than 100 years, and to clear the lineage in the inheritance process.

The intangible cultural heritage and national feature of life are closely related, fully displaying the national life personality and aesthetic standards, is human centered and exist, and to form cultural and artistic interpretation, as a continuation of the intangible cultural heritage, so the inheritance is the most important. In the traditional clothing design, many non-material cultural elements are worthy of our heritage, and its application in the modern fashion design process, will inevitably inject new vitality to the clothing design. 


\section{The traditional hand collar of Hanfu clothing and dance}

First we to two ancient costume design as an example, which has a long history of clothing and clothing, it is Jiangxi and even the Chinese national intangible cultural heritage. Hanfu in the design process, the left and right collar collar stand up, and across his chest, formed in liling. If to analyze this kind of design with traditional culture, the show is a positive atmosphere, that person or partial positive, the harmony between man and nature, man to founder, has a profound meaning, shows the China culture.

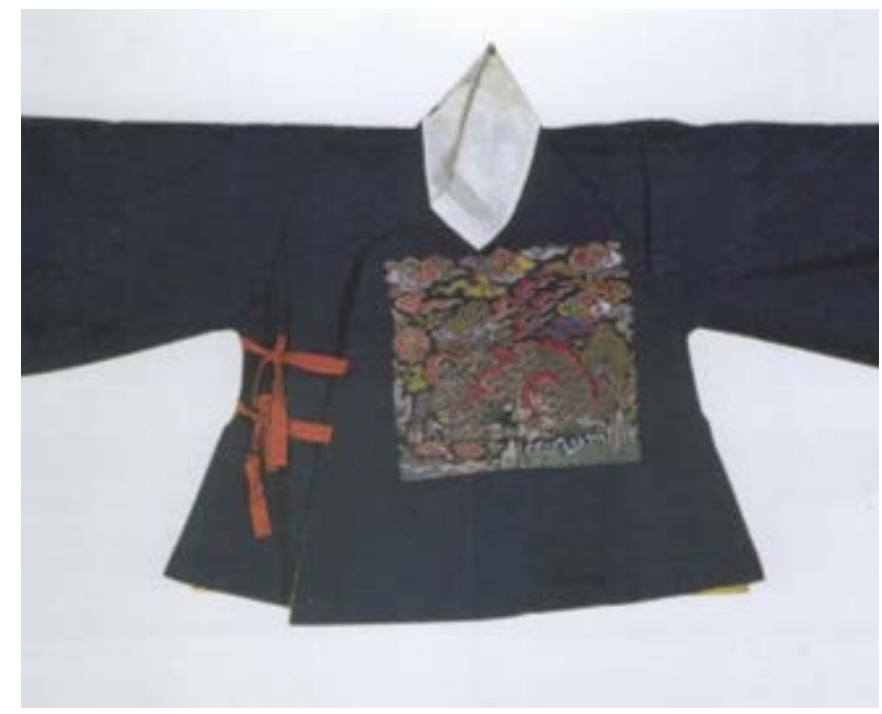

Fig.1 The hand collar of hanfu

In the development process also appeared in Liling, significantly improved, as shown in Figure 1, the emergence of the "Jiaoling Youren" is directly connected with the lapel collar, lapel cross in the chest, right on the left side of the skirt pressed skirts showed "Y" shape in appearance. This is mainly to the right collar collar, this design style and ancient to the right to close the idea of respecting, this shows that the elements of traditional culture has been integrated into the clothing design, similarly there Jiangxi Nanfeng Nuo dance clothing cross collar design.

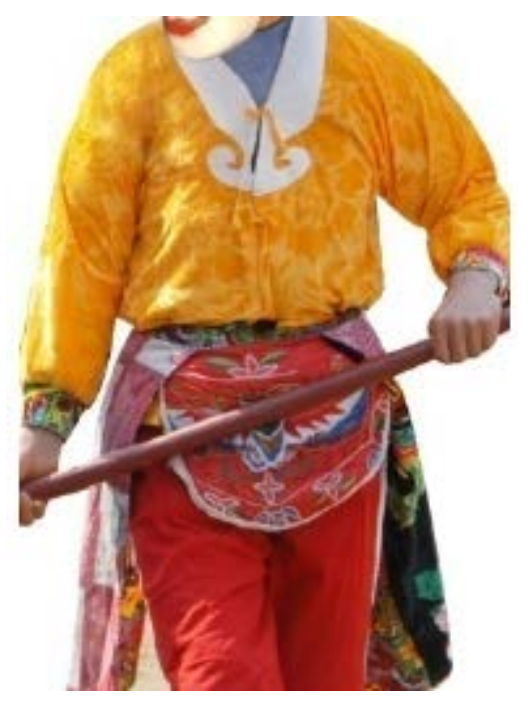

Fig.2 The hand collar of dance clothing

According to the investigation of scholars Wang Hanyun, in the clothing also have cross collar dance clothing, the clothing is a kind of Nuo dance performed in general, there are two children wearing the clothing of deduction, a design of the cross collar is also very popular in the current. 


\section{The inspiration of the inheritance of the intangible cultural heritage to the modern fashion design}

In recent years, with the Hanfu and clothing as a prototype to exorcise Liling clothing gradually prevailed, the collar is a core part of fashion design, fashion designers are at all times and in all countries attach great importance to the collar design, the neckline is the clothing highlights, can foil a person's neck and neck and face in Hanfu clothing design process of the ancient Nuo dance this factor can be considered. The orthodox Hanfu cross collar version is generally the sleeve, straight, before and after the raphe, clear the Chinese elements in shape generally closer to Hanfu, but will change the sleeve through the sleeve, and appropriate waist design, as a prototype, clothes designed by simple and elegant, as shown in Figure 3.

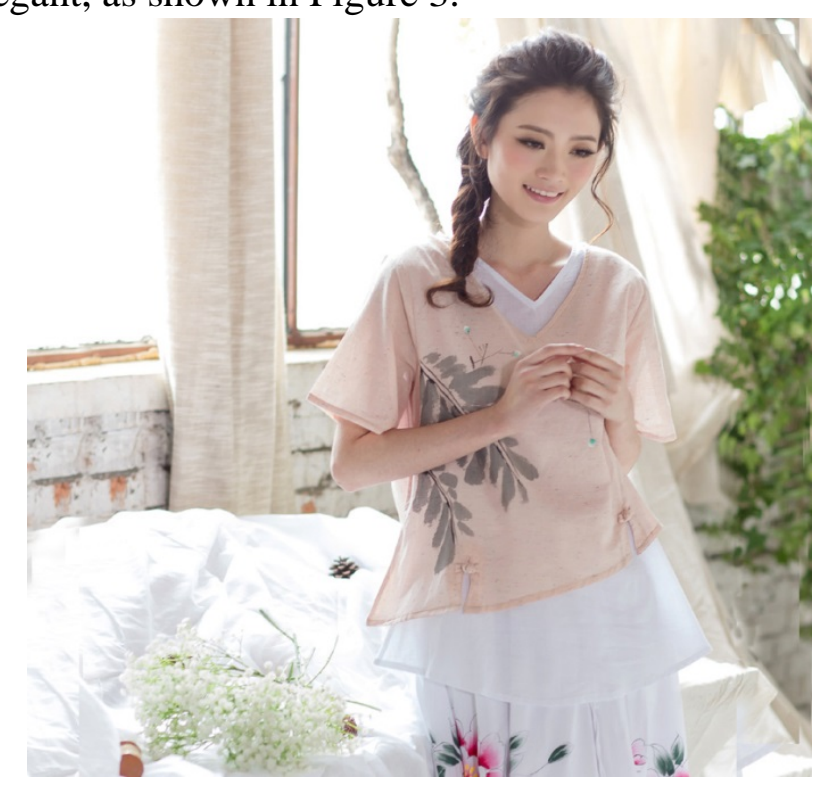

Fig.3 The application of turn collar in modern clothing design

As shown in Figure 3, uses Liling design clothing to women's gentle leisure simple, elegant, showing the charm of Oriental women. While the more common and daily will be brought in most of the elements into the fashion design and variety to keep Hanfu version, but if the cultural elements of Hanfu clothing is also far into the design, will be designed to bring new ideas, and can be generalized and inheritance of the intangible heritage of the Chinese nation. Also in the costume design, the non-material culture is not lost at the same time, the clothing has broader prospect.

\section{Conclusion}

Jiangxi intangible cultural heritage of many cultural elements can be used in modern fashion design, fashion design in the modern fashion popular, often become cross collar designers use their brains, they often use the characteristics of the cross collar handsome, graceful, convey new feelings from different angles, or narrowing or widening. The design is the use of modern decoration materials, techniques, or change the right to left, however, these characteristics were derived from the design inspiration and clothing collar to exorcise hanfu. The combination of Hanfu and clothing collar to Nuo dance and modern design and new cloth depth, can emit China belongs to the modern clothing charm.

\section{Reference}

[1] Liu Yueping. Preliminary study on the design of clothing exhibition space[J]. Guangdong architectural decoration, 2007, (04). 
[2] Li Hongqin. The modern design direction of folk costume. [J]. Anhui Literature (second half), 2006, (09).

[3], Chen Peizhao, Wang Xihui. Interpretation of cultural connotation of Hua Yao costume[J]. Journal of Chengde Nationalities Teachers College, 2006, (02).

[4], Sun Yun. An analysis of the significance of combining Chinese ethnic costume culture with modern fashion design [J]. Journal of Taiyuan City Vocational College, 2007 (06).

[5], Zhu Jianbo. Investigation of the characteristics and folk custom of Korean traditional costumes [J]. Journal of Northeast Dianli University, 2006. (05).

[6] Yin smile. Floating like cloud Chinese ancient costume beauty straightening if scared dragon [J]. Oriental Art. 2006, (02) .

[7] Lin Chaoyang. Aesthetic sense of Chinese ancient costume color [J]. Journal of Donghua University (SOCIAL SCIENCE EDITION), 2005, (03).

[8] Xia, clothing CAD application. The advantages and disadvantages of the study [J]. Journal of Yulin Normal University, 2012 (05).

[9]. Zhao Baosheng. Excel Application skills[J]. Digital Technology and Applications,2012 (07).

[10] Sun Li. Application and existing problems of clothing CAD. [J]. Public Technology,2010 (11).

[11] He Guiliang, Dai Hong. Study on current situation and development of CAD/CAM usage in clothing industry [J]. Hebei textile,2008 (02).

[12] Zhu Guangzhou. Popularization and application of clothing [J].CAD knitting industry,2007 (08).

[13] Zhang Qize, Huang Fang. Analysis of development and application of domestic clothing CAD technology [J]. Guangxi textile technology,2007 (01).

[14], Jiao Huiqin, Feng Wei Yi, Zhang Yunpeng. Research on the application of SVG in clothing design system [J].Silk, 2007 (01).

[15] Dong Ge. Study on the defect texture in fabric design [J]. Art and Design (Theory),2009 (08).

[16] Wang Mei, Lu Guodong, Zhang Dongliang. Research and application of 3D human modeling technology in garment [J]. Journal of engineering graphics,2007 (01).

[17] Ke Huiling. Art fashion renderings[J]. Art\&life,2006 (05).

[18] Teng Xiaobo, Su bin. From New Media Art to Information Art- Interview with Prof. Lu Xiaobo[J]. Decoration,2004 (12).

[19] Wang Qiming. Analysis of the methods and current situation of 3D human body measurement in apparel industry [J]. Journal of Shaoxing University (NATURAL SCIENCE),2002 (10) . 